\title{
Conducting a Literature Review
}

The literature review is an integral part of any research project and is undertaken as a means of surveying what research has been conducted previously on a particular topic. There are many reasons for conducting a literature review, but one of the primary reasons is to establish a base line of what is already known on a topic before exploring the topic any further. The review typically involves a search of any previously published or presented materials that might have relevance to a prospective new study.

\section{Original Research vs. Survey of the Literature}

If the point of a research project is solely to review what has already been written on a topic, the resulting article is termed a "survey of the literature" or a "literature survey" or even a "literature review." In this case, the article

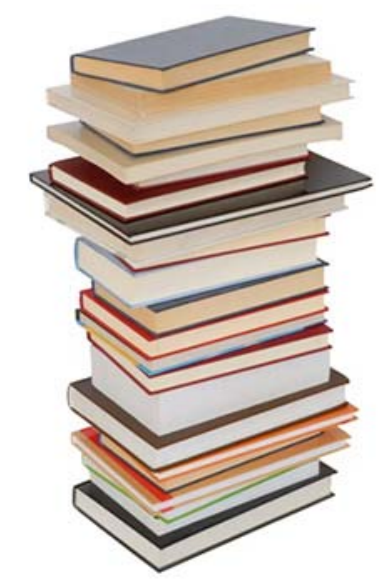
is complete in itself and does not delve into anything new regarding the topic. A literature survey might end with a discussion of what work is still needed to further develop knowledge of a particular topic, but it does not, itself, flesh out any of those ideas. Articles of this type can be highly beneficial to someone seeking to launch an original study; literature surveys have already laid some of the groundwork for a prospective researcher's own literature review.

When the survey serves as the initial step that precedes a further investigation of an idea or ideas about a topic, then that review of the literature sets the stage for the presentation of original research. Original research usually involves the selection of a methodology for examining a topic and may include the gathering of data that can be further analyzed to arrive at assumptions about the topic. Data may be derived from the examination of human subjects, from conducting surveys or assessments, from the study of particular species of plants or animals, from the systematic scientific measurement of any physical phenomena, from nearly anything that can be documented and analyzed. Again, the whole point of launching an original study is to learn something new about a topic. Research typically begins with what is known (the literature review) and progresses into analyzing, through the observation and analysis of data, what is yet to be known through further study.

Both the literature survey and the original study are considered academic articles, as opposed to popular articles. Both involve research in order to come to a better understanding of a topic.

\section{Benefits of Conducting a Literature Review}

While there might be many reasons for conducting a literature review, following are four key outcomes of doing the review.

Assessment of the current state of research on a topic. This is probably the most obvious value of the literature review. Once a researcher has determined an area to work with for a research project, a search of relevant information sources will help determine what is already known about the topic and how extensively the topic has already been researched. 
Identification of the experts on a particular topic. One of the additional benefits derived from doing the literature review is that it will quickly reveal which researchers have written the most on a particular topic and are, therefore, probably the experts on the topic. Someone who has written twenty articles on a topic or on related topics is more than likely more knowledgeable than someone who has written a single article. This same writer will likely turn up as a reference in most of the other articles written on the same topic. From the number of articles written by the author and the number of times the writer has been cited by other authors, a researcher will be able to assume that the particular author is an expert in the area and, thus, a key resource for consultation in the current research to be undertaken.

Identification of key questions about a topic that need further research. In many cases a researcher might discover new angles that need further exploration by reviewing what has already been written on a topic. For example, research might suggest that listening to music while studying might lead to better retention of ideas, but the research might not have assessed whether a particular style of music is more beneficial than another. A researcher who is interested in pursuing this topic would then do well to follow up existing studies with a new study, based on previous research, that tries to identify which styles of music are most beneficial to retention.

Determination of methodologies used in past studies of the same or similar topics. It is often useful to review the types of studies that previous researchers have launched as a means of determining what approaches might be of most benefit in further developing a topic. By the same token, a review of previously conducted studies might lend itself to researchers determining a new angle for approaching research.

Upon completion of the literature review, a researcher should have a solid foundation of knowledge in the area and a good feel for the direction any new research should take. Should any additional questions arise during the course of the research the researcher will know which experts to consult in order to quickly clear up those questions.

\section{Steps in Conducting a Literature Review}

Conducting a literature review involves using research databases to identify materials that cover or are related in some sense to the research topic. In some cases the research topic might be so original in its scope that no one has done anything exactly like it, so research that is at least similar or related will provide source material for the literature review. The selection of databases will be driven by the subject matter and the scope of the project.

Selecting Databases -- Most academic libraries now provide access to a majority of their databases and their catalog via a so-called discovery tool. A discovery tool makes searching library systems more "Google-like" in that even the simplest of queries can be entered and results retrieved. However, many times the results are also "Google-like" in the sheer quantity of items retrieved. While a discovery tool can be invaluable for quickly finding a multitude of resources on nearly any topic, there are a number of considerations a researcher should keep in mind when using a discovery tool, especially for the researcher who is attempting a comprehensive literature review.

No discovery tool works with every database subscribed to by a library. Some libraries might subscribe to two or three hundred different research databases covering a large number of subject areas. Competing discovery systems might negotiate agreements with different database vendors in order to provide access to a large range of materials. There will be other vendors with whom agreements are not forthcoming, therefore their materials are not included in the discovery tool results. While this might be of only minor concern for a researcher looking to do a fairly limited research project, the researcher 
looking to do a comprehensive review of the literature in preparation for writing a master's thesis or a doctoral dissertation will run the risk of missing some materials by limiting the search just to a particular library's discovery system. If only one system covered everything that a researcher could possibly need, libraries would have no need to subscribe to hundreds of different databases. The reality is that no one tool does it all. Not even Google Scholar.

Book collections might be excluded from results delivered by a discovery tool. While many libraries are making results from their own catalogs available via their discovery tools, they might not cover books that are discoverable from other library collections, thus making a search of book collections incomplete. Most libraries subscribe to an international database of library catalogs known as WorldCat. This database will provide comprehensive coverage of books, media, and other physical library materials available in libraries worldwide.

Features available in a particular database might not be available in a discovery tool. Keep in mind that a discovery tool is a search system that enables searching across content from numerous individual databases. An individual database might have search features that cannot be provided through a discovery tool, since the discovery tool is designed to accommodate a large number of systems with a single search. For example, the nursing database CINAHL includes the ability to limit a search to specific practice areas, to limit to evidence-based practice, to limit to gender, and to search using medical subject headings, among other things, all specialized facets that are not available in a discovery tool. To have these advanced capabilities, a researcher would need to go directly to CINAHL and search it natively.

Some discovery tools are set, by default, to limit search results to those items directly available through a particular library's collections. While many researchers will be most concerned with what is immediately available to them at their own library, a researcher concerned with finding everything that has been done on a particular topic will need to go beyond what's available at his or her home library and include materials that are available elsewhere. Master's and doctoral candidates should take care to notice if their library's discovery tool automatically limits to available materials and broaden the scope to include ALL materials, not just those available.

With the foregoing in mind, a researcher might start a search by using the library's discovery tool and then follow up by reviewing which databases have been included in the search and, more importantly, which databases have not been included. Most libraries will facilitate locating its individual databases through a subject arrangement of some kind. Once those databases that are not discoverable have been identified, the researcher would do well to search them individually to find out if other materials can be identified outside of the discovery tool. One additional tool that a doctoral researcher should of necessity include in a search is ISI's Web of Knowledge. The two major systems searchable within ISI's $W e b$ are the Social Sciences Citation Index and the Science Citation Index. The purpose of these two systems is to enable a researcher to determine what research has been cited over the years by any number of researchers and how many times it has been cited.

Formulating an Effective Search Strategy -- Key to performing an effective literature review is selecting search terms that will effectively identify materials that are relevant to the research topic. An initial strategy for selecting search terminology might be to list all possible relevant terms and their synonyms in order to have a working vocabulary for use in the research databases. While an individual subject database will likely use a "controlled vocabulary" to index articles and other materials that are included in the database, the same vocabulary might not be as effective in a database that focuses on a different subject area. For example, terminology that is used frequently in psychological literature might not be as effective in searching a human resources management database. Brainstorming the topic before 
launching into a search will help a researcher arrive at a good working vocabulary to use when probing the databases for relevant literature.

As materials are identified with the initial search, the researcher will want to keep track of other terminology that could be of use in performing additional searches. Sometimes the most effective search terminology can be found by reading the abstracts of relevant materials located through a library's research databases. For example, an initial search on the concept of "mainstreaming" might lead the researcher to articles that discuss mainstreaming but which also look into the concept of "inclusion" in education. While the terms mainstreaming and inclusion are sometimes used synonymously, they really embody two different approaches to working with students having special needs. Abstracts of articles located in the initial search on mainstreaming will uncover related concepts such as inclusion and help a researcher develop a better, more effective vocabulary for fleshing out the literature review.

In addition to searching using key concepts aligned with the research topic, a researcher likely also will want to search for additional materials produced by key authors who are identified in the initial searches. As a researcher reviews items retrieved in the initial stages of the survey, he or she will begin to notice certain authors coming up over and over in relation to the topic. To make sure that no stone is left unturned, it would be advisable to search the available, relevant library databases for other materials by those key authors, just to make sure something of importance has not been missed. A review of the reference lists for each of the items identified in the search will also help to identify key literature that should be reviewed.

Locating the Materials and Composing the Review -- In many cases the items identified through the library's databases will also be available online through the same or related databases. This, however, is not always the case. When materials are not available online, the researcher should check the library's physical collections (print, media, etc.) to determine if the items are available in the library, itself. For those materials not physically available in the home library, the researcher will use interlibrary loan to procure copies from other libraries or services. While abstracts are extremely useful in identifying the right types of materials, they are no substitute for the actual items, themselves. The thorough researcher will make sure that all the key literature has been retrieved and read thoroughly before proceeding too far with the original research.

The end result of the literature review is a discussion of the central themes in the research and an overview of the significant studies located by the researcher. This discussion serves as the lead section of a paper or article that reports the findings of an original research study and sets the stage for presentation of the original study by providing a review of research that has been conducted prior to the current study. As the researcher conducts his or her own study, other relevant materials might enter into the professional literature. It is the researcher's responsibility to update the literature review with newly released information prior to completing his or her own study.

Updating the Initial Search -- Most research projects will take place over a period of time and are not completed in the short term. Especially in the case of master's and doctoral projects, the research process might take a year or several years to complete. During this time, it will be important for the researcher to periodically review the research that has been going on at the same time as his or her own research. Revisiting the search strategies employed in the initial pass of the literature will turn up any new studies that might have come to light since the initial search. Fortunately, most research databases and discovery systems provide researchers with the means for automatically notifying them when new materials matching the search strategy have entered the system. This requires that a researcher sign up for a personal "account" with the database in order to save his or her searches and set up "alerts" when 
new materials come online. Setting up an account does not involve charges to the researcher; this is all a part of the cost borne by the home library in providing access to the databases.

A Summary of the Process -- The following summarizes the steps in conducting a literature review.

- Identify appropriate library databases for conducting the research. This might begin with the library discovery tool.

- Identify relevant search terms for use in conducting a database search.

- Review initial search results, review article abstracts, and revise search strategy and terms accordingly.

- Look for key concepts that appear in the literature and identify significant authors who are frequently cited in the literature.

- Conduct a new search for materials by the key authors identified in the previous step.

- Locate the materials revealed by the literature review and read and digest.

- Construct a written overview of the major concepts revealed by a thorough reading of the literature. How does this material relate to the current study?

- Once the original research is completed, do a follow-up review of the literature to determine if there is anything significant that should also be included.

Reading by Jim Alderman. Updated March 2014. 